\title{
Custos em pomares de pessegueiro conduzidos nos sistemas integrado e convencional, nos municípios de Araucária e Lapa, Paraná
}

\author{
Peaches orchard costs assessment produced in integrated and conventional systems in \\ municipalities of Araucária and Lapa, Paraná
}

\author{
Joel Ferreira Penteado Junior ${ }^{I^{*}}$ Louise Larissa May De Mio \\ Honorino Roque Rodigheri ${ }^{\mathrm{I}}$
}

RESUMO

Este trabalho teve como objetivo o cálculo e a análise de custos nos sistemas de produção integrada (PI) $e$ convencional $(P C)$ de pêssego, em dois estabelecimentos agrícolas localizados nos municípios de Araucária e Lapa, Paraná. Foram apropriados os custos de produção baseados nas atividades. Os coeficientes técnicos e os indicadores foram empregados por hectare e por ano, sendo relativos à utilização de insumos ao longo de três anos, contemplando: quantidade dos direcionadores de custo, custo unitário da atividade, percentual de participação dos coeficientes na atividade e os gastos de cada componente dos custos, divididos esses em grandes grupos como: serviços, insumos, outros insumos e administrativos. Os custos totais por hectare nos pomares da PI, nos três anos, foram 5,86 e 5,05\% inferiores ao custo da $P C$, respectivamente para Araucária e Lapa, PR. Os itens mais representativos na composição dos custos foram os gastos com mão-de-obra e agrotóxicos.

Palavras-chave: custos de produção, avaliação econômica, sistemas de produção.

\section{ABSTRACT}

This research aimed to calculate and analysis the costs of integrated system (IP) and conventional (PC) peaches production in two agricultural establishments located in the Araucária and Lapa counties - Paraná. The cost production based activities were appropriate and technical coefficients and indicators were related to the use of inputs over three years, employed per hectare and by year Management cost parameters were included, such as: estimating target cost, activity unit cost, percentage of participation in the activity of the coefficients and expenses involved in each component of the cost structure. It was divided into large groups, such as services, supplies, administrative and other supplies. The total cost per hectare in the IP orchards of IP were $5.86 \%$ and
5.05\% lower than the PC cost respectively for Araucária and Lapa, PR. The items most representative in the cost composition were spent with the workforce and pesticides.

Key words: production costs, economic evaluation, production systems.

\section{INTRODUÇÃO}

A exploração racional dos pomares de pêssegos depende de um conjunto de fatores que podem afetar o desempenho produtivo e a rentabilidade, tais como manejo e relação de preços entre insumos e produto (MEDEIROS, 1998). A implantação e manutenção exigem altos investimentos, além de os cultivos apresentarem, normalmente, retornos financeiros significativos somente a partir do quinto ano (EMBRAPA, 2006). Devido a essa característica, o conhecimento dos custos e dos sistemas de produção de pêssegos passam a ser importantes na tomada de decisão para a otimização dos fatores de produção (MADAIL et al., 2002).

No Brasil, a fruticultura gera um PIB de US\$ 1,5 bilhão (EMBRAPA, 2006) e, entre as fruteiras de clima temperado, a cultura do pessegueiro é uma das mais importantes, com produção de 130.000 toneladas ao ano. Os Estados do Sul são os maiores produtores de pêssego do país, onde as condições naturais favorecem a exploração comercial (IBGE, 2006). No Paraná, o município de Lapa é o maior produtor dessa

IEmpresa Brasileira de Pesquisa Agropecuária (Embrapa Florestas). Estrada da Ribeira, Km 111, 80411-000, Colombo, PR, Brasil. E-mail:joel@cnpf.embrapa.br. *Autor para correspondência.

IUniversidade Federal do Paraná (UFPR). Rua dos Funcionários, nº 1540, 80035-050, Curitiba, PR, Brasil. E-mail: maydemio@ufpr.br. 
fruta, com produção anual de 5.040t, e Araucária é o segundo, com uma produção anual de 940 mil t (IPARDES, 2006). Foi também nesse município que se iniciou, em 2001, a Produção Integrada (PI) de pêssegos (FACHINELLO et al., 2003), sistema de produção que objetiva, principalmente, estabelecer uma relação de confiança para o consumidor, garantindo que o produto está conforme os requisitos determinados nas Normas Técnicas Específicas (ANDRIGUETO \& KOSOSKI, 2005).

As frutas oriundas da PI podem possibilitar vantagens de mercado. No entanto, existem dúvidas quanto ao impacto da adoção desse sistema na rentabilidade do pomar. Essas dúvidas decorrem, principalmente, do desconhecimento do comportamento dos custos nesse sistema de produção (FARIAS et al., 2004). Um dos melhores métodos de apropriação é o "custo por atividades" (DI DOMENICO, 1995), fundamentado num processo de acumulação e rastreamento de dados sobre as atividades, o que, dessa forma, auxilia e direciona a empresa nas decisões operacionais (BRIMSON, 1991).

O presente trabalho objetivou apresentar cálculos e fazer a análise dos custos de produção em pomares de pessegueiro que adotam a PI, comparandoos com o sistema de Produção Convencional (PC), nos municípios de Araucária e Lapa, PR.

\section{MATERIAL E MÉTODOS}

Caracterização dos estabelecimentos agrícolas

Em Araucária, o estudo foi conduzido em dois pomares de pessegueiros, onde o estabelecimento agrícola dispõe de nível tecnológico superior e conta com recursos para investimento e infra-estrutura para produção, beneficiamento e armazenamento. Conta, ainda, com possibilidade de acesso à diversidade de mercados para comercialização. A área total da propriedade é de 217 hectares, sendo que a atividade principal é a produção de grãos. A produção de pêssego e ameixa, apesar de utilizarem apenas $8 \%$ da área, representa aproximadamente $50 \%$ do faturamento total do estabelecimento.

Em Lapa, o estabelecimento agrícola possui área total de 12 hectares, onde são cultivados pessegueiro, ameixeira e nectarineira. Esse estabelecimento dispõe também de bom nível tecnológico, mas não tem acesso aos mesmos canais de distribuição do estabelecimento de Araucária.

Nos dois estabelecimentos agrícolas, as áreas foram manejadas nos ciclos vegetativos de 2002, 2003 e 2004, em dois sistemas de produção, sendo um conduzido de acordo com as Normas Técnicas para PI
- NTPI (MAPA, 2003) e outro no sistema PC, no qual as práticas culturais e o manejo fitossanitário são aqueles normalmente utilizados pelo produtor, como: tratamentos fitossanitários com calendário fixo, sem monitoramento de pragas e manejo realizado conforme critério próprio.

Apropriação dos custos e coleta dos dados Os dados contabilizam a utilização de insumos ao longo de três anos, representados pelos coeficientes e pela quantidade empregada por hectare e por ano, contemplando os gastos envolvidos em cada componente da estrutura de custos, divididos em alguns grandes grupos, assim distribuídos: a) Serviços, em que estão discriminados os custos com mão-deobra e mecanização; b) Insumos, em que são contemplados os gastos necessários para o controle de doenças, pragas, preparo do solo e manejo; c) Outros insumos, envolvendo gastos com caixas e sacolas de colheita; análise foliar e de solo; mudas; herbicidas; armadilhas, atraentes e feromônios para monitoramento de insetos e d) Administrativos, que incluem as despesas administrativas, impostos e as realizadas com manutenção e depreciação de máquinas e equipamentos utilizados exclusivamente na implantação do pomar (Tabela 1 ).

\section{RESULTADOS E DISCUSSÃO}

Custos de Produção em Araucária e Lapa, PR.

Os resultados encontrados na apropriação dos custos na PI e na PC, em Araucária, demonstram que os custos totais, no período de 2002 a 2004, foram de $\mathrm{R} \$ 13.518,06$ ha $^{-1}$ para o pomar da PC e de R $\$ 12.726,75$ ha $^{-1}$ para o pomar da PI, representando uma diferença menor no sistema PI de 5,86\%.

Quanto ao estabelecimento agrícola de Lapa, os dispêndios também são maiores na PC, totalizando R\$9.779,85 ha-1, enquanto que, na PI, os gastos são de $\mathrm{R} \$ 9.256,37 \mathrm{ha}^{-1}$, proporcionando uma diferença 5,05\% maior na PC, quando comparada à PI.

Apesar de os preços dos insumos serem semelhantes nos dois locais estudados, os custos de produção variaram em função da diferença entre os níveis de adoção tecnológica e disponibilidade de recursos humanos e financeiros dos dois estabelecimentos. A diferença entre as características de solo, topografia e manejo também foram responsáveis pelas variações nos custos.

Em valores totais, os gastos no estabelecimento agrícola de Araucária são maiores que os de Lapa, e os resultados apresentados por VICENZI (2003), comparando custos em pomares de pêssegos 
Tabela 1 - Custos totais da produção (R\$/ha/ano) e percentagem de gastos em relação ao custo total nos sistemas de produção integrada (PI) e convencional (PC) de pêssego, em Lapa, PR, nos anos de 2002 a 2004.

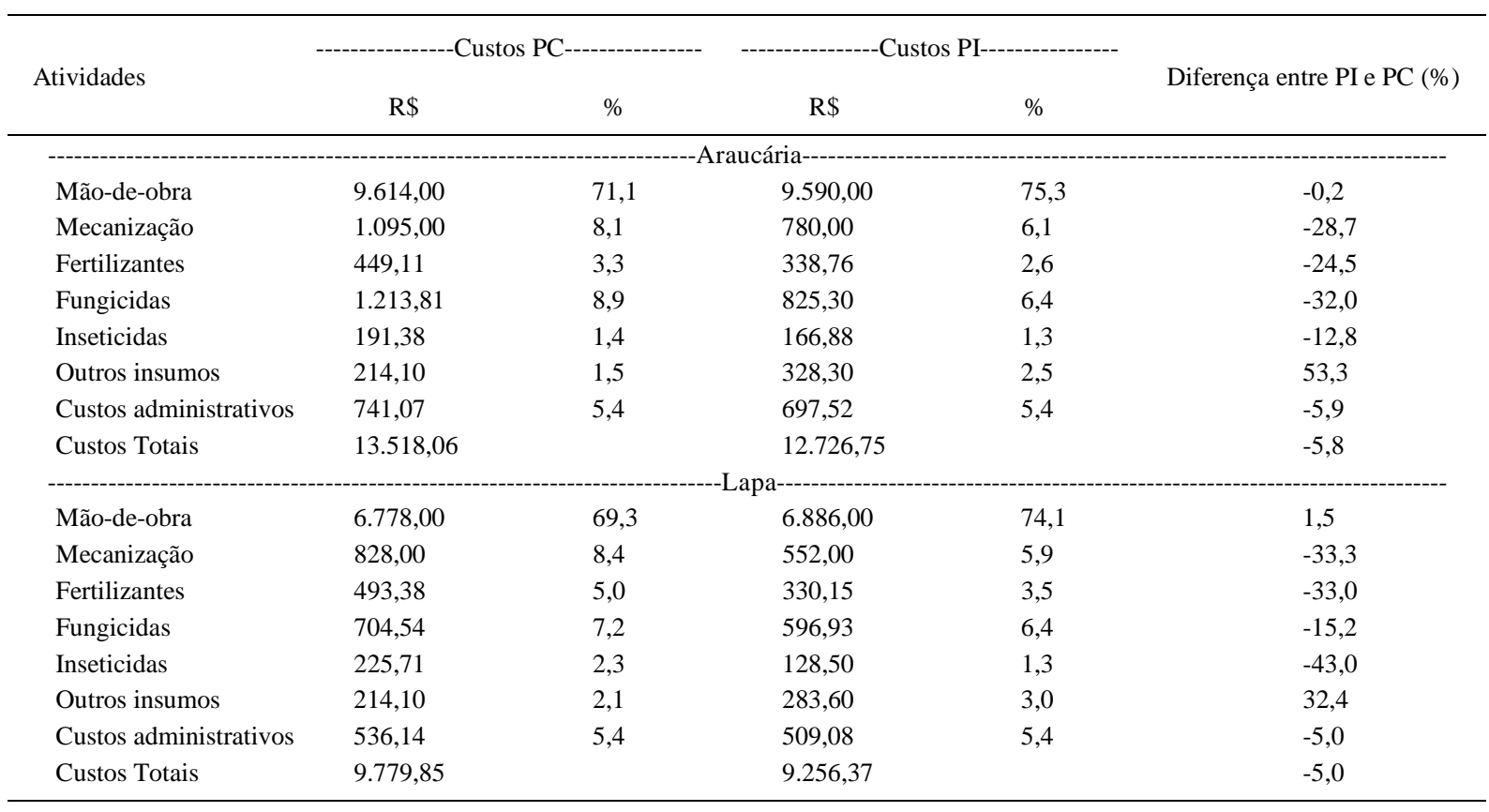

no Rio Grande do Sul, demonstram que o custo de produção na PI foi 14,78\% superior ao da PC. A redução média dos custos entre os estabelecimentos de Araucária e Lapa é de R\$1.314,79 ha-1 . Portanto, se essa média se repetir em todos os pomares de pêssego que aderiram à produção integrada no Brasil, o que totaliza 520ha (IBGE, 2006), isso proporcionaria uma economia de $\mathrm{R} \$ 683.690,80$ para os produtores de pêssegos.

\section{Custos de Serviços}

Na tabela 1, foram discriminados os custos anuais de "serviços". Especificamente com mão-deobra, em Araucária, o valor total aplicado do quinto ao sétimo ano foi de R $\$ 9.614,00 \mathrm{ha}^{-1}$, no sistema PC, contra $\mathrm{R} \$ 9.590,00$ ha $^{-1}$ no PI, indicando uma diferença de $0,25 \%$ a menos no sistema PI. A atividade de roçada entre as linhas e capina manual, característica requerida pelo sistema PI, foi um dos pontos de maior diferença entre os sistemas. No entanto, devido à menor produtividade da PI, no estabelecimento agrícola utilizado para esta pesquisa, as despesas de mão-de-obra com colheita e raleio foram maiores que à da PC. A participação dos gastos com mão-de-obra em relação ao custo total representou $71,12 \%$ na PC e $75,35 \%$ na PI, demonstrando que o peso percentual da mão-de-obra é maior no sistema PI.

Quanto às atividades desenvolvidas no estabelecimento de Lapa (Tabela 1), os custos de serviços com mão-de-obra no sistema PC foram de
R \$6.778,00 ha-1 e no PI de R \$6.886,00 ha ${ }^{-1}$, apresentando também um gasto maior no PI, porém de apenas 1,59\%. As atividades desenvolvidas com poda, raleio e colheita foram as que demandaram os maiores aportes de recursos financeiros. As atividades desenvolvidas com capinas manuais e roçadas nas entrelinhas apresentaram as maiores diferenças entre os sistemas. Os custos adicionais dessas atividades, aplicados nos pomares PI, são justificados devido à utilização mais rigorosa e intensiva da mão-de-obra na execução das práticas preconizadas pelas normas da produção integrada. O comportamento dos gastos com serviços de mão-de-obra é semelhante ao encontrado por FARIAS et al. (2004), em produção de pêssego na região central do Rio Grande do Sul, confirmado essa característica do sistema PI.

O uso intensivo de mão-de-obra na fruticultura é uma característica da atividade. Conforme estudos de MADAIL et al. (2002), o item serviços, representado pela mão-de-obra, foi o que alcançou a maior participação no custo em produção convencional de pêssegos em estudos no Rio Grande do Sul, totalizando $60,80 \%$. Segundo ARAÚJO \& CORREIA (2004), o custo total com mão-de-obra para as culturas de graviola, goiaba, acerola, maracujá e abacaxi, representaram 40,79, 44,41, 50,55, 58,25 e 56,18\%, respectivamente.

Quanto à composição dos custos com mecanização em Araucária (Tabela 1), na PC, o valor foi 
de R $\$ 1.095,00 \mathrm{ha}^{-1}$, e, na PI, foi de R $\$ 780,00 \mathrm{ha}^{-1}$. Para o estabelecimento de Lapa (Tabela 1), os valores foram de $\mathrm{R} \$ 828,00$ ha $^{-1}$ para o sistema PC e de $\mathrm{R} \$ 552,00$ para o PI, indicando gastos 33,33\% maiores no sistema PC, em comparação ao PI. Os custos maiores com mecanização no sistema PC se devem ao fato de que o sistema PI reduz as atividades com tratores, como redução no número de roçadas e de uso de grade ou enxada rotativa.

Os valores gastos com mecanização nos pomares localizados nos dois estabelecimentos agrícolas e também nos dois diferentes sistemas de produção foram inferiores aos contabilizados pela EMBRAPA (2006) em PC de pêssego, no Estado do Rio Grande do Sul, onde o valor foi de R $\$ 1.456,00 \mathrm{ha}^{-1}$, devido a maiores necessidades de aplicação de agrotóxicos naquele pomar.

\section{Custos de Insumos}

Em relação aos fertilizantes, verifica-se que os valores gastos no pomar de Araucária (Tabela 1) foram de R \$449,11 ha-1 na PC e de R \$338,76 ha ${ }^{-1}$ na PI, apresentando uma diferença $24,50 \%$ menor na PI. Quanto ao estabelecimento agrícola de Lapa (Tabela 1), os valores gastos foram de R\$493,38 na PC e R \$330,15 na PI, apresentando uma diferença 33,08\% maior na PC. Em ambos os estabelecimentos, as adubações na PI seguiram as recomendações da NTPI (MAPA, 2003) e, na área da PC, seguiram a praticada normalmente pelos produtores. Portanto, a redução nos custos é reflexo direto das instruções preconizadas pela PI.

Os resultados demonstrados neste estudo são semelhantes aos apresentados por Farias et al. (2004), que obtiveram valores de R $\$ 252,00$ na PC e R \$120,00 na PI, em avaliação de custos para a mesma espécie e com nível tecnológico semelhante no Estado do Rio Grande do Sul. Quanto aos valores gastos com fungicidas, no estabelecimento de Araucária (Tabela 1), o custo foi maior na PC, quando comparado à PI, ou seja, de R $\$ 1.213,81$ ha $^{-1}$ para os três anos na PC e de R\$825,30ha- ${ }^{-1}$ na PI; portanto, 32,01\% a menos na PI.

No estabelecimento agrícola de Lapa (Tabela 1 ), os valores gastos com fungicidas foram de $\mathrm{R} \$ 704,54 \mathrm{ha}^{-1}$ no sistema PC e de $\mathrm{R} \$ 596,93 \mathrm{ha}^{-1}$ no sistema PI, com uma diferença 15,27\% menor no sistema PI, em relação ao PC.

Na safra de 2002, ou seja, no sexto ano de vida do pomar, foram feitas 20 pulverizações de fungicidas na PI, contra 30 na PC; e, no sétimo ano, foram feitas 17 na PI, contra 22 na PC (MAY-DE MIO et al., 2004).

Os valores obtidos neste estudo são semelhantes às observações de ANDRIGUETO \&
KOSOSKI (2005), os quais verificaram que a redução de aplicação de fungicidas em pomares de pêssego no sistema PI foi $20 \%$ menor em relação ao PC. Segundo ALMEIDA et al. (2007), em estudos de produção integrada de abacaxi no Estado de Tocantins, a redução com fungicida propiciada pelo sistema PI foi de $47 \%$.

Em relação aos gastos realizados com inseticidas em Araucária (Tabela 1), a PC foi mais dispendiosa, custando $\mathrm{R} \$ 191,38 \mathrm{ha}^{-1}$, contra $\mathrm{R} \$ 166,88 \mathrm{ha}^{-1}$ da PI. Assim, o custo com a PI foi $12,80 \%$ menor que com a PC.

Em Lapa, a PI também apresentou dispêndios menores com inseticidas, quando comparada à PC. Os valores totais foram de R $\$ 225,71 \mathrm{ha}^{-1}$ na PC e R $\$ 128,50$ ha $^{-1}$ na PI. A diferença percentual foi 43,06\% menor na PI.

Na PI, a tendência é que se diminua o número de aplicações de inseticidas, pois as plantas concorrentes, se bem manejadas, são importantes aliadas para o manejo do solo e das pragas, uma vez que servem de abrigo aos inimigos naturais, e a convivência positiva com a vegetação torna-se de grande importância para o pomar (FARIAS, 2004).

Segundo MOLINARI (2001), o produtor que adotar o sistema de PI irá aplicar agrotóxicos somente nos períodos recomendados, ou quando as pragas atingirem o nível de controle, o que significa uma redução no número de aplicações. De acordo com SANSAVINI (1998), GRELLMANN et al. (1991), FACHINELLO et al. (2003) e FARIAS et al. (2004), o uso indiscriminado de agrotóxicos por parte dos agricultores tem preocupado alguns segmentos da sociedade, não só quanto ao aspecto de poluição ambiental, mas também quanto à qualidade da fruta. Assim, deve-se priorizar a utilização de produtos eficientes para o controle da praga em questão, e com menor impacto sobre o meio ambiente, pois, além de promover redução nos custos, há o benefício ambiental.

Custos de Outros Insumos

Os dispêndios com “outros insumos” para o estabelecimento agrícola de Araucária (Tabela 1) foram 53,34\% maiores no sistema PI, com valor de R $\$ 328,30 \mathrm{ha}^{-1}$, contra R $\$ 214,10$ ha $^{-1}$ gastos na PC. No estabelecimento de Lapa (Tabela 1), o comportamento dos gastos foi semelhante ao de Araucária, PR, apresentando valores maiores na PI. Na PC, o dispêndio foi de R $\$ 214,10$ ha $^{-1}$ e, na PI, foi de R $\$ 283,60 \mathrm{ha}^{-1}$.

Os valores maiores nesse caso se devem a gastos com a utilização de armadilhas para insetos, atrativos e feromônios adotados pela PI e que não são utilizados na PC. No entanto, houve redução nos gastos 
com herbicidas nos dois estabelecimentos estudados. Em Araucária, a redução foi de 33,58\% e, em Lapa, foi de $66,60 \%$. Ambos os estabelecimentos estudados apresentaram dados semelhantes aos de FARIAS et al. (2004), que, em uma análise de pomares de pessegueiros em São Jerônimo, RS, constataram que, na PC, foram utilizados cinco litros de herbicida, no período de uma safra, e, na PI, foram utilizadas apenas roçadas manuais nas entrelinhas.

\section{Custos Administrativos}

Os valores dos custos administrativos (Tabela 1) corresponderam a 5,48\% dos custos totais na PC e na PI. Os métodos para apropriação de custos administrativos de trabalhos disponíveis na literatura diferem entre si, dificultando a sua comparação. Nos trabalhos de custos de produção de pêssego de MADAIL et al. (2002) e FARIAS et al. (2004), não são contabilizados os custos administrativos. Nos trabalhos de MATSUNAGA et al. (1976) e MARTIN et al. (1998), que servem de referência para a grande maioria dos trabalhos de avaliação econômica de culturas agrícolas, os custos administrativos correspondem, em média, a $5 \%$ dos custos totais.

Em uma análise conjunta dos dados relativos à comparação de custos, aponta-se uma redução média com o gasto com agrotóxicos entre os dois estabelecimentos agrícolas estudados de $\mathrm{R} \$ 512,26 \mathrm{ha}^{-1}$. Considerando-se que a área total de PI de pêssego no Brasil é de 520ha (IBGE, 2006), a redução nos custos com esses insumos seria de R $\$ 266.375,20$. Esse valor corresponde a uma redução no volume de: 700 litros de inseticidas, 728 litros de fungicidas, $47.320 \mathrm{~kg}$ de fertilizantes e 828 litros de herbicidas.

Dessa forma, a PI possibilita um melhor equilíbrio ecológico, favorecendo o bem-estar humano e a recuperação de organismos benéficos, que são importantes aliados na luta biológica e na viabilidade de um sistema de produção menos dependente de insumos químicos (SANSAVINI, 1998).

\section{CONCLUSÕES}

A apropriação dos custos pode variar de acordo com o nível tecnológico e as práticas culturais adotadas nos diferentes estabelecimentos agrícolas.

O sistema de produção integrada em pomares de pêssego propicia redução nos custos de produção. Em Araucária, a redução dos custos foi de 5,86\% e, em Lapa, foi de 5,05\%.

\section{REFERÊNCIAS}

ALMEIDA, C.O. et al. Avaliação de impactos da produção integrada de abacaxi no estado do Tocantins: um estudo de caso de um sistema de transição. Cruz das Almas: Embrapa Mandioca e Fruticultura Tropical, 2007. 29p. (Embrapa Mandioca e Fruticultura Tropical. Documentos, 167).

ANDRIGUETO, J.R.; KOSOSKI, A.R. Desenvolvimento e conquistas da produção integrada de frutas no Brasil até 2004. Relatório 2005. Brasília: Ministério da Agricultura, Pecuária e Abastecimento, 2005. 10p.

ARAÚJO, J.L.P.; CORREIA R.C. Avaliação dos impactos ambientais do sistema de produção de manga na Região do Submédio São Francisco. In: SEMINÁRIO BRASILEIRO DE PRODUÇÃO INTEGRADA DE FRUTAS, 2004, Petrolina, PE. Anais... Petrolina-PE: Embrapa Semi-Árido/Embrapa Uva e Vinho/Embrapa Agroindústria Tropical/Embrapa Meio Ambiente, Valexport, 2004

BRIMSON, J.A. Activity accounting: an activity-based costing approach. New York: John \& Sons, 1991. 214p.

DI DOMENICO G.B.; LIMA, P.C. Gestão de custos baseada em atividades em um ambiente agrícola. In: CONGRESSO INTERNACIONAL DE CUSTOS, 1995, Campinas, SP. Anais... Campinas: Universidade Estadual de Campinas, 1995. p.407-419.

EMPRESA BRASILEIRA DE PESQUISA AGROPECUÁRIA. Sistema de produção de pêssego. Disponível: w w w . c n p u v. e m b r a p a . b r / p u b l i c a / s p r o d / PessegodeMesaRegiaoSerraGaucha/custos.htm. Acesso em: 15 nov 2006.

FACHINELLO, J.C. et al. Normas técnicas e documentos de acompanhamento da produção integrada de pêssego. Pelotas: Universidade Federal de Pelotas, Faculdade de Agronomia Eliseu Maciel, 2003. 95p.

FARIAS, R.M. et al. Produção convencional x integrada em pessegueiro cv. Marli na Depressão Central do Rio Grande do Sul. Revista Brasileira de Fruticultura, Jaboticabal, v.25, n.2, p.253-255, 2004.

GRELLMANN, E.O et al. Exigências térmicas e estimativa do número de gerações de Grapholita molesta (Busck,1916) (Lepidoptera: Olethreutidae) em Pelotas, RS. 1991. 42f. Dissertação (Mestrado em Ciências Biológicas) - Universidade Federal de Pelotas, Pelotas, RS.

IBGE de Recuperação Automática. Desenvolvido pelo Instituo Brasileiro de Geografia e Estatística. Apresenta informações estatísticas. Disponível em: www.ibge.gov.br. Acesso em jul 2006.

IPARDES - INSTITUTO PARANAENSE DE DESENVOLVIMENTO ECONOMICO E SOCIAL. Caderno Estatístico, Município de Lapa. Cadernos Municipais Ipardes 2006. (b) Disponível em w w w. i p a r d e s. g o v. b r / c a d e r n o s/ Montapdf.php?Municipio=83750\&btOk=ok. Acesso em 10 ago 2006.

MADAIL, J.C.M. et al. Análise da rentabilidade dos sistemas empresarial e familiar de produção de pêssego no sul do Rio Grande do Sul. Pelotas: Embrapa Clima 
Temperado, 2002. 43p. (Embrapa Clima Temperado. Documentos, 86).

MEDEIROS, A.R.M. Leguminosas de inverno: uma opção no manejo do solo em pomar de fruteiras de clima temperado. Hortisul, Pelotas, v.2, n.1, p.14-15, 1998.

MAPA - Ministério da Agricultura e Pecuária e Abastecimento. Estatística/agronegócio brasileiro 2003. Disponível em: <http.www.agricultura.gov.br> Acesso em: 15 ago 2007.

MARTIN, N.B. et al. Sistema Integrado de Custos Agropecuários - CUSTAGRI. Informações Econômicas, São Paulo, v.28, n.1, 101p. 1998.

MATSUNAGA, M. et al. Metodologia do custo de produção utilizada pelo IEA. Agricultura em São Paulo, São Paulo, v.23, n.1, p.123-139, 1976.

MAY-DE MIO, L.L. et al. Comparação entre os sistemas convencional e integrado para produção de pêssegos em
Araucária-PR. In: CONGRESSO BRASILEIRO DE FRUTICULTURA, 2004, Florianópolis, SC. Anais... Florianópolis: SBF, 2004. CD ROM.

MOLINARI, F. La difesa dal fitofagi nella produzione integrata del pesco in Itália. In: SEMINÁRIO SOBRE PRODUÇÃO INTEGRADA DE FRUTAS, 3., 2001, Bento Gonçalves, RS. Anais... Bento Gonçalves: Embrapa Uva e Vinho. 2001. p.4858

SANSAVINI, S. Integrated fruit production: process, issues, prospects after ten years' experience. In: CONGRESSO BRASILEIRO DE FRUTICULTURA, 15, 1998, Poços de Caldas. Anais... Lavras, MG: UFLA, 1998. p.133-135.

VICENZI, M. Avaliação econômica de dois sistemas de produção de pêssego (Prunus persica L. Batsch): Convencional (PC) $x$ Integrada (PI) no Município de Pelotas. 2003. 47f. Dissertação (Mestrado em Fruticultura) - Faculdade de Agronomia. Universidade Federal de Pelotas, RS. 